\title{
Recommendations for the use of bioresorbable vascular scaffolds in percutaneous coronary interventions
}

\author{
B. Everaert ${ }^{1,12} \cdot$ J. J. Wykrzykowska ${ }^{2} \cdot$ J. Koolen ${ }^{3} \cdot$ P. van der Harst ${ }^{4} \cdot$ P. den Heijer ${ }^{5}$ J. P. Henriques ${ }^{2} \cdot$ R. van \\ der Schaaf $^{6}$ B. de Smet $^{7}$ - S. H. Hofma ${ }^{8}$ R. Diletti ${ }^{1}$ A. Weevers ${ }^{9} \cdot$ J. Hoorntje $^{10}$ • P. Smits ${ }^{11}$ • R. J. van Geuns ${ }^{1}$
}

Published online: 22 June 2017

(C) The Author(s) 2017. This article is an open access publication.

\begin{abstract}
Background To eliminate some of the potential late limitations of permanent metallic stents, the bioresorbable coronary stents or 'bioresorbable vascular scaffolds' (BVS) have been developed.

Methods We reviewed all currently available clinical data on BVS implantation.

Results Since the 2015 position statement on the appropriateness of BVS in percutaneous coronary interventions, several large randomised trials have been presented. These have demonstrated that achieving adequate 1 and 2 year outcomes with these first-generation BVS is not straightforward. These first adequately powered studies in noncomplex lesions showed worse results if standard implan-
\end{abstract}

R. J. van Geuns

r.vangeuns@erasmusmc.nl

1 Thoraxcenter, Erasmus Medical Centre, Rotterdam, The Netherlands

2 Academic Medical Center, Amsterdam, The Netherlands

3 Catharina Hospital, Eindhoven, The Netherlands

4 University Medical Center, University of Groningen, Groningen, The Netherlands

5 Amphia Hospital, Breda, The Netherlands

6 Onze Lieve Vrouwe Gasthuis, Amsterdam, The Netherlands

7 Meander Medical Center, Amersfoort, The Netherlands

8 Medical Center, Leeuwarden, The Netherlands

9 Albert Schweitzer Hospital, Dordrecht, The Netherlands

10 Maastricht University Medical Center, Maastricht, The Netherlands

11 Maasstad Hospital, Rotterdam, The Netherlands

12 Monica Hospital, Antwerp, Belgium tation techniques were used for these relatively thick scaffolds. Post-hoc analyses hypothesise that outcomes similar to current drug-eluting stents are still possible if aggressive lesion preparation, adequate sizing and high-pressure postdilatation are implemented rigorously. As long as this has not been confirmed in prospective studies the usage should be restricted to experienced centres with continuous outcome monitoring. For more complex lesions, results are even more disappointing and usage should be discouraged. When developed, newer generation scaffolds with thinner struts or faster resorption rates are expected to improve outcomes. In the meantime prolonged dual antiplatelet therapy (DAPT, beyond one year) is recommended in an individualised approach for patients treated with current generation BVS.

Conclusion The new 2017 recommendations downgrade and limit the use of the current BVS to experienced centres within dedicated registries using the updated implantation protocol and advise the prolonged usage of DAPT. In line with these recommendations the manufacturer does not supply devices to the hospitals without such registries in place.

Keywords Bioresorbable vascular scaffold P Percutaneous coronary intervention · Absorb BVS

\section{Introduction}

Drug-eluting stents (DES) are widely used and considered first choice devices in percutaneous coronary interventions (PCI) to treat ischaemic coronary artery disease [1]. These permanent implants, however, do not have any residual function after vascular healing following PCI. Beyond the initial healing period, metallic stents may induce new problems, resulting in an average reintervention rate of $2 \%$ per 
year [2]. To eliminate some of the potential late limitations of permanent metallic DES, such as impaired vasomotion, hampered endothelial function, reduced potential for vessel remodelling and interference with future non-invasive imaging (cardiac computed tomography or magnetic resonance imaging) or treatment modalities (re-PCI or coronary artery bypass grafting), bioresorbable coronary stents or 'bioresorbable vascular scaffolds' (BVS) have been developed. As clinical evidence is accumulating, we have updated the 2015 Dutch recommendations on the use of BVS in PCI [3].

\section{Lesion selection}

The short-term efficacy of BVS in patients with non-complex coronary lesions has been investigated and reported in four large independent randomised trials (ABSORB II, ABSORB China, ABSORB Japan and ABSORB III [4-7]). The characteristics of these 'Absorb 2/3-like lesions' are summarised in Table 1.

At 1 year, Absorb BVS (Abbott Vascular, Santa Clara, CA) were demonstrated to be non-inferior to Abbott's Xience DES regarding the combined and individual clinical endpoints of death, myocardial infarction (MI), and target lesion failure/revascularisation. These results were confirmed in a meta-analysis of randomised clinical trial data at 1 year, comprising 3389 patients randomised in a 2:1 fashion to Absorb BVS and Xience DES, respectively [8]. However, a signal of higher incidence of target vessel MI was reported, partly due to a non-significant nominal increase in device thrombosis (hazard ration (HR) 2.09). In the longer term, the 5-year results of the ABSORB cohort B trial were encouraging, showing late lumen stability and the restoration of vasomotor function, together with low restenosis and major adverse cardiac event (MACE) rates in relatively simple stenotic lesions [9]. This was in line with expectations regarding a potential long-term physiological

Table 1 BVS Absorb 2/3-like lesions

\begin{tabular}{ll}
\hline Absorb 2/3-like lesions & Exclusion \\
\hline De novo lesions & Left main \\
Diameter 2.3-3.8 mm & Arterial or venous grafts \\
Maximum length $28 \mathrm{~mm}$ & In-stent restenosis \\
One BVS scaffold overlap & Chronic total occlusion \\
Maximum 2 lesions & Ostial lesions \\
Stable, unstable or silent & Bifurcation lesions with side branches \\
ischaemia & $\geq 2$ mm diameter \\
- & Excessive calcification \\
- & High tortuosity \\
- & Visible thrombus \\
- & (N)STEMI \\
- & LVEF <30\% \\
\hline
\end{tabular}

benefit after full bioresorption of scaffolds over permanent metallic DES. However, after 3 years, the ABSORB II study did not report any superiority in vasomotor reactivity of BVS over DES [10]. Moreover, the device-oriented composite endpoint was significantly worse in the Absorb BVS subgroup (HR 2.17), mainly due an increase in target vessel MI (6\% vs. $1 \% ; p=0.011)$.

Furthermore, also the 2 year results from the ABSORB III study were reported [11] and the rates of target lesion failure were significantly higher with BVS than DES ( $11.0 \%$ vs. $7.9 \%, p=0.03)$, mainly driven by an increase in target vessel MI, which was partly due to an increase in the incidence of scaffold thrombosis (ScT).

In the initial ABSORB trials, the implantation technique was reported to be suboptimal according to current BVS implantation standards. In a pooled analysis (ABSORB II, ABSORB China, ABSORB Japan and ABSORB III and ABSORB Extend) optimal sizing, optimal sizing/predilatation and optimal sizing/pre- and postdilatation was only achieved in about $81.6 \%, 59.2 \%$ and $12.4 \%$ of patients, respectively [12]. In particular optimal sizing avoiding implantation in vessels with a reference vessel diameter (RVD) by quantitative coronary analysis (QCA) of $<2.25 \mathrm{~mm}$ or $>3.5 \mathrm{~mm}$ reduced both 2 year target lesion failure $(12.1 \%$ vs. $7.6 \%, p=0.0006)$ and stent thrombosis (2.6\% vs. $1.5 \%, p=\mathrm{ns})$ [13]. In a blinded, pooled interim analysis of ABSORB IV, the rate of ScT at 30 days and after 1 year was lower compared with ABSORB III ( $0.3 \%$ vs. $1.0 \%$ and $0.5 \%$ vs. $1.3 \%$, respectively), which was attributed to higher adherence to improved implantation techniques, in particular avoidance of implantation in vessels with an RVD $<2.25$ (4\% vs. 19\%, respectively) and applying proper post-dilatation (83\% vs. 66\%, respectively). Still we will have to wait for the completion of this trial to see the unblinded data.

Given the lack of clear benefit on mid-term outcomes compared with contemporary DES with repeated signals of safety issues regarding early, late and possibly very late stent thrombosis, we have to downgrade the level of recommendation for 'Absorb 2/3-like lesions' from 'appropriate' to 'potentially appropriate' and restricted to experienced centres with continuous safety monitoring within dedicated registries using the most recent implantation technique (as described in the instructions for use of the device). Patient should be well informed on the potential risks of the therapy and the only hypothetical long-term benefit.

For patients with more complex lesions, who were excluded from the aforementioned Absorb 2/3 studies, the mid-term outcome data of some medium-sized studies on more complex, real-world lesions and indications have been reported. For instance, the BVS Expand Registry, a single-centre registry including a more complex patient and lesion subset, showed a clinical device success of $97.3 \%$ 


\section{Advertisement placed here.}

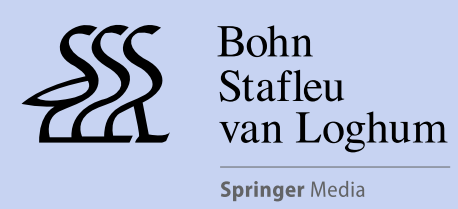

Houten 2017 


\section{Advertisement placed here.}

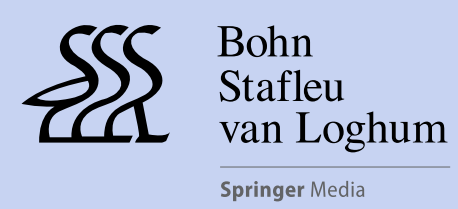

Houten 2017 
and a MACE rate of $6.8 \%$ at 18 months [14]. Secondly, from a large real-world BVS registry (GHOST-EU) the 1 -year clinical result data were compared with propensitymatched patients from a large post-marketing DES registry (Xience V USA). No differences in target lesion failure, target vessel MI or stent/scaffold thrombosis were noticed [15].

Recently, the Amsterdam Investigator-initiateD Absorb Strategy all-comers trial (AIDA) trial, an investigator-initiated, randomised non-inferiority trial in the context of routine clinical practice, was published early because of safety concerns raised by the study data and safety monitoring board (median duration of follow-up of 707 days) [16]. This study included much more complex patients compared with the company initiated Absorb phase 3 studies requirements for approval. In AIDA, 54\% of the patients presented with acute coronary syndrome (ACS), which was not allowed in the Absorb studies. A large number of complex lesions were included (5\% bifurcations, $4 \%$ chronic total occlusions (CTO), 5.6\% ostial and 30\% moderately or severely calcified) with on average longer lesions and more scaffolds per patient and per lesion. Still there was no significant difference regarding the primary endpoint of target vessel failure at 2 years $(11.7 \%$ vs. $10.7 \%)$ nor in the secondary endpoints of target vessel and target lesion revascularisation $(8.7 \%$ vs. $7.5 \%, 7.0 \%$ vs. $5.2 \%$, respectively). Most importantly, there was a highly significant difference in the rates of definite and probable ScT at 2 years $(3.5 \%$ with BVS vs. $0.9 \%$ with metallic DES, $p<0.001)$. This was reflected in a higher rate of target vessel MI (5.5\% vs. $3.2 \%$, $p=0.04)$. A more detailed analysis showed, besides an increase in early and late ScT, also the occurrence of very late ScT. For this reason, the data and safety monitoring board recommended early reporting of the study to inform the patients and physicians. Concerning the implantation technique, the use of postdilatation was higher compared with previous studies (74\%) but not yet at the current target level and as in many other studies, small vessels (reference vessel diameter $\leq 2.25 \mathrm{~mm}$ by QCA) accounted for almost $20 \%$ of the lesions. Vessel size of $\leq 2.25 \mathrm{~mm}$, adequate device sizing or postdilatation did not seem to be not associated with the occurrence of ScT; however, the relatively small sample size (31 ScT cases) might have limited this analysis. This contrasts with the findings from a casecontrol analysis of 105 ScTs occurring in randomised controlled trials (RCTs) and large registries (>200 BVS-treated patients) with at least 12 months of follow-up [17], in which early dual antiplatelet therapy (DAPT) cessation, no-postdilatation and RVD $<2.4 \mathrm{~mm}$ were identified as predictors for ScT.

The GHOST-EU registry included 302 bifurcation lesions. The rate of target lesion failure with BVS was acceptable (6.4\% at 360 days). However, the rates of ScT were elevated (2.5\%) [18]. For long coronary lesions ( $\geq 60 \mathrm{~mm})$, target lesion failure rate with BVS was substantially higher ( $14.3 \%$ compared with $4.8 \%$ and $4.5 \%$ for lesions $<30 \mathrm{~mm}$ or between 30 and $60 \mathrm{~mm}$, respectively), mainly because of an increase in MI (including ScT (3.8\%)) and clinically driven target lesion revascularisation [19]. Of note, scaffold overlapping did not appear to have a negative impact on a composite endpoint of all-cause death, any MI and any repeated revascularisation as well as on the rate of early or late ScT [20]. Concerning BVS implantation in ACS patients (ST-segment elevation myocardial infarction (STEMI), non-STEMI, unstable angina) the rates of patientand device-oriented endpoints, as well as ScT, were significantly elevated compared with non-ACS patients [21]. One multicentre, single-blinded, RCT reported on BVS use in primary PCI in patients with STEMI. In the TROFI II study, arterial healing with BVS implantation was comparable to DES and nearly complete at 6 months and target lesion revascularisation at 6 months was low in both study arms (1.1\% vs. 0\%) [22]. Regarding CTOs, a recent study on complex CTO (all lesions with J-CTO score $>2$, mean 2.61) reported procedural success rates of $97.1 \%$ and favourable mid-term results at up to 6 months with 3 MACE in 105 patients $(2.9 \%)$ [23]. We have now included this group of lesions in the general more complex group. Still, longer follow-up is needed to draw more definitive conclusions on BVS use in both STEMI and CTO lesions.

In summary, the new data on BVS use in more complex lesions for up to 2 years is more concerning compared with our previous analysis and we changed the previous advice from probably appropriate to discouraged for routine clinical practice.

The data on 'highly' complex lesions, such as two scaffold bifurcations, severely calcified lesions and aorta-ostial lesions, however, are still premature or not in favour of BVS use. As such, for these highly complex lesions the use of BVS is currently not supported by expert opinion.

Furthermore, for two subsets of lesions, namely arterial or venous grafts and in-stent restenosis, the current Absorb BVS label (de novo lesions in native vessels) does not apply and the off-label use of BVS should be extraordinary.

A final - technical - limitation is the overexpansion capabilities of the Absorb BVS that is currently restricted to $0.5 \mathrm{~mm}$ based on the recommendation of the manufacturer. As the largest commercially available Absorb BVS is $3.5 \mathrm{~mm}$ at nominal pressure, vessels with a diameter above $4.0 \mathrm{~mm}$ should not be targeted because of the risk of extensive malapposition. We do not support the implantation of BVS in lesions with an RVD below $2.25 \mathrm{~mm}$ (as measured by QCA) as the outcome in this lesion subgroup is significantly worse as reported by the Absorb III trial investigators (target lesion failure: $12.9 \%$ (Absorb BVS) vs. $8.3 \%$ (Xience DES)) [5]. Another caveat is ostial coronary 
Table 2 Lesion selection
Absorb 2/3-like lesions: 'de novo' lesions, max. length $28 \mathrm{~mm}$, one stent overlap, max. 2 lesions, RVD $>2.25 \mathrm{~mm}$ on QCA optimal implantation technique (PSP)

Discouraged for routine clinical practice $^{\mathrm{a}}$

Use not supported by data or expert opinion

Not recommended

ACS patients, including STEMI

Long lesions ( $>28 \mathrm{~mm},<60 \mathrm{~mm}$ ) (diameter stenosis $<40 \%$ after pre-dilatation)

Provisional bifurcation treatment (including fenestration of the side branch)

Non-complex CTO (J-CTO score $<2$ )

Complex CTO (J-CTO score $\geq 2$ )

Very long lesions $(\geq 60 \mathrm{~mm})$

Ostial coronary lesions

Severely calcified lesions with failure to prepare properly

Bifurcation lesions requiring a two scaffold approach

RVD $<2.5$ ( $2.25 \mathrm{~mm}$ on QCA)
Moderately to severely calcified lesions with proper lesion preparation

In-stent restenosis ${ }^{\mathrm{b}}$

Arterial and venous grafts ${ }^{\mathrm{b}}$

Vessel $>4.0 \mathrm{~mm}$ in diameter ${ }^{\mathrm{b}}$

$A C S$ acute coronary syndrome, $C T O$ chronic total occlusion, $P S P$ pre-dilate, size properly and post-dilate, $Q C A$ quantitative coronary analysis, $R V D$ reference vessel diameter, STEMI ST-elevation myocardial infarction

${ }^{\mathrm{a} U}$ Use restricted to dedicated BVS clinical trial/registries

${ }^{\mathrm{b}}$ Off-label use

Table 3 Patient selection

\begin{tabular}{|c|c|c|}
\hline Optimal & Patient with good life expectancy (i. e. $>5$ years) & $\begin{array}{l}\text { Age }<70 \text { years or Age } 70-80 \text { with a maximum of } 1 \text { of: } \\
\text { severe renal failure or dialysis, DM, BMI }>40 \text { or LVEF } \\
<40 \% \text {, stroke, PAD or COPD }\end{array}$ \\
\hline $\begin{array}{l}\text { No potential benefit to be } \\
\text { expected }\end{array}$ & $\begin{array}{l}\text { Patient with limited life expectancy (i.e. } \\
<2-3 \text { years) }\end{array}$ & $\begin{array}{l}\text { Cardiogenic shock, severe heart failure }(\mathrm{EF}<30 \%) \text {, dialy- } \\
\text { sis }\end{array}$ \\
\hline Avoid & $\begin{array}{l}\text { No use in emergency bail-out situations } \\
\text { Patients on oral anticoagulants }\end{array}$ & - \\
\hline
\end{tabular}

$B M I$ body mass index, $C O P D$ obstructive pulmonary disease, $D M$ diabetes mellitus, $L V E F$ left ventricular ejection fraction, $P A D$ peripheral artery disease

lesions, as was suggested by data from the GHOST-EU registry [24]. The composite endpoint of a combination of cardiovascular death, target vessel MI or target lesion revascularisation was significantly higher in ostial lesions $(12.6 \%$ vs. $4.6 \%$ ) as was the incidence of ScT ( $4.9 \%$ vs. $2.0 \%)$. The use of BVS in this group is not supported by sufficient data, and the authors of this consensus statement advise to avoid use in these lesions until more data are available.

Table 2 provides the advice of the authors on lesion characteristics used for identification of possible target lesions as descripted above.

\section{Patient selection}

As bioresorbable scaffolds resolve 2-3 years after implantation, improvement in patient outcomes in comparison with permanent metallic structures, if present, will probably be most evident in patients whose life expectancy exceeds those first years of implantation.
It is therefore essential to appropriately select patients in which BVS may yield the highest advantage on longtime clinical outcome compared with DES. The ideal BVS candidate is a young first time presenter with a good life expectancy ( $>5$ years). On the other hand, patients above 80 years, patients with severe renal failure or on dialysis and patients who are in cardiogenic shock at the time of the implantation only have a limited life expectancy [25] and therefore the potential for a long-term benefit of BVS compared with DES is severely hampered. Other patient-related conditions, such as diabetes mellitus, body mass index $>40$, left ventricular ejection fraction $<40 \%$, previous stroke, peripheral artery disease and chronic obstructive pulmonary disease, also have a negative impact on patient's life expectancy and should be carefully weighted in terms of risks and benefits. In Table 3 we summarise the patient characteristics that can be useful for patient selection. The use of BVS in emergency bailout situations is currently not supported. Also BVS implantation in patients on oral anticoagulants should be avoided because of the need of longterm DAPT ( $\geq 3$ years) due to the risk of very late ScT. 


\section{Technical considerations for BVS implantation}

We want to stress that an optimal BVS implantation technique will be of paramount importance for obtaining good long-term clinical results [24]. Lesion preparation is especially important, as, before inflation, the initial scaffold diameter is quite large $(1.4 \mathrm{~mm})$ which is related to the specific scaffold-related folding characteristics of the Absorb BVS. Therefore, highly calcified or tortuous lesions or lesions with a high degree of angulation can be quite challenging for BVS implantation. However, with extensive lesion pre-dilatation using increasing balloon sizes, even calcified lesions can be successfully treated with BVS, although special care has to be paid to a good implantation technique. In summary, we advise the 'P-S-P' implantation technique:

1. 'Prepare the lesion' aggressively using adequate predilatation (1:1 vessel-to-balloon ratio), We caution not to implant BVS in lesions were predilatation balloons do not fully expand.

2. 'Size the vessel/scaffold correctly'. On-line QCA or preferably invasive imaging (intravascular ultrasound [IVUS] and optical coherence tomography [OCT]) is advisable in general and indispensible in small vessels (reference vessel diameter $<3 \mathrm{~mm}$ or using a $2.5 \mathrm{~mm}$ BVS scaffold). Evidently, undersizing has to be avoided and never implant if the RVD is below $2.5 \mathrm{~mm}$ (measured by invasive imaging).

3. 'Post-dilate' the scaffold with a properly sized non-compliant balloon to avoid underexpansion (using a noncompliant balloon with a diameter at least equal to and preferably $0.5 \mathrm{~mm}$ larger than the RVD using high inflation pressures [18-20 atm]).

Also, be aware of the expansion limits of the implanted BVS as overexpansion of the scaffold can potentially lead to scaffold fractures (maximal $0.5 \mathrm{~mm}$ larger than scaffold diameter). The effect of a BVS specific implantation strategy was deduced from a large registry covering four hospitals and $>1300$ patients. After implementation of a specific BVS implantation protocol, incidence of ScT was significantly reduced (3.3\% vs. $1.0 \%$ at 12 months for suboptimal vs. optimal, respectively) [26]. In this retrospective analysis failure to achieve a final minimal lumen diameter of $2.5 \mathrm{~mm}$ for the small design (nominal $2.5 \mathrm{~mm}$ or $3.0 \mathrm{~mm}$ devices) or $2.9 \mathrm{~mm}$ for the larger design (nominal $3.5 \mathrm{~mm}$ devices) was an important factor in the occurrence of ScT.

To avoid BVS malapposition, correct scaffold sizing based on a reliable assessment of vessel dimensions is a second important issue. Invasive imaging modalities, such as IVUS and OCT, can be of great value in providing accurate morphometry for estimating vessel diameter and lesion length in circumstances where angiographic assessment is ambiguous. OCT is particularly suited to visualise the apposition of scaffold struts to the vessel wall and can guide BVS optimisation [27]. Before implantation, OCT is indicated to predetermine lesion characteristics, such as vessel diameter, lesion length and the amount of calcification, to estimate the optimal scaffold length and to identify the optimal proximal and distal landing zones. The importance of appropriate vessel sizing is becoming increasingly clear, and, as already mentioned, BVS implantation in vessels with a diameter of less than $2.25 \mathrm{~mm}$ on quantitative coronary imaging (comparable with a diameter of less than $2.5 \mathrm{~mm}$ on visual estimation) should be avoided because of substantially worse clinical outcomes and an elevated risk of ScT [4]. OCT after scaffold implantation can be a helpful tool to guide postdilatation of the scaffold with properly sized non-compliant balloons to optimise strut apposition, taking into account the expansion limit of $0.5 \mathrm{~mm}$ for the Absorb BVS.

\section{Antiplatelet therapy}

DAPT has been the basis for minimising stent thrombosis for many years. Current guidelines for antiplatelet therapy advise DAPT for 6-12 months in stable angina patients receiving DES [28]. For ACS patients, based on the European Society of Cardiology non-STEMI and STEMI guidelines a minimum of 12 months of DAPT is advised, preferably with prasugrel or ticagrelor [29, 30]. More recent publications suggest that for a low-risk population and when using second generation DES, DAPT duration might be shortened to 6 months $[31,32]$. With the longer follow-up period of larger registries and RCTs very late ScT has emerged as a major problem. Timewise this might be related to the resorption process of the BVS. Although in preclinical settings this has never been noticed as a problem, the current hypothesis is related to the expected degradation and disintegration of devices without full embedment in the vessel wall. Areas of malapposed, disintegrating struts might become instable and trigger thrombosis. This might be an additional risk factor to other already identified risk factors for very late stent thrombosis.

The Dual Antiplatelet Therapy (DAPT) study demonstrated that in patients who tolerated DAPT after DES implantation for 12 months, without a bleeding complication, DAPT up to 30 months, as compared with aspirin therapy alone, significantly reduced the risks of stent thrombosis and major adverse cardiovascular and cerebrovascular events but was associated with an increased risk of bleeding [33]. Within this very large $(n=11,648)$ randomised study with 348 ischaemic events, multiple risk factors were identified ([34]; Table 4) demonstrating the multifactorial process of stent thrombosis, one of which was the use of 
Table 4 Predictors of myocardial infarction or stent thrombosis $12-30$ months post-PCI in the DAPT study

\begin{tabular}{llc}
\hline Predictors of MI or ST & HR $(95 \%$ CI $)$ & $P$ value \\
\hline MI at presentation & $1.65(1.31-2.07)$ & $<0.001$ \\
Prior PCI or prior myocardial infarction & $1.79(1.43-2.23)$ & $<0.001$ \\
History of CHF or LVEF $<30 \%$ & $1.88(1.35-2.62)$ & $<0.001$ \\
Vein graft stent & $1.75(1.13-2.73)$ & 0.01 \\
Stent diameter $<3 \mathrm{~mm}$ & $1.61(1.30-1.99)$ & $<0.001$ \\
Paclitaxel-eluting stent & $1.57(1.26-1.97)$ & $<0.001$ \\
Diabetes mellitus & $1.38(1.10-1.72)$ & 0.01 \\
Peripheral arterial disease & $1.49(1.05-2.13)$ & 0.03 \\
Hypertension & $1.37(1.03-1.82)$ & 0.03 \\
Renal insufficiency/failure & $1.55(1.03-2.32)$ & 0.04 \\
\hline
\end{tabular}

$C H F$ congestive heart failure, $L V E F$ left ventricular ejection fraction, $M I$ myocardial infarction, $P C I$ percutaneous coronary intervention, $S T$ stent thrombosis paclitaxel-eluting stents (with thick struts). Finally, a model was developed balancing the increased bleeding risk of prolonged DAPT duration with the reduction in ischaemic events (DAPT score).

Recently, the PEGASUS-TIMI 54 trial [35] evaluated long-term therapy with ticagrelor in addition to aspirin, in patients with a history of spontaneous MI occurring 1-3 years prior to randomisation. The PEGASUS study concluded that ticagrelor significantly reduced the risk of MACE as compared with placebo. Recently, two meta-analyses [36, 37] showed that DAPT beyond 1 year among stabilised high-risk patients with prior MI decreased ischaemic events at the cost of an increase in major bleeding. Udell et al. showed that prolonged DAPT $>1$ year could reduce the rate of stent thrombosis [36].

For the Absorb BVS a minimum of 6 months DAPT is required per protocol (ABSORB-EXTEND, ABSORB II), and the majority of patients in most studies received DAPT for 12 months. Because of signals of a higher occurrence of early as well as late, and even very late stent thrombosis (beyond 1 year), currently and until further confirmation, the best advice is to prescribe DAPT for a minimum of 3 years for BVS Absorb implanted patients in a tailored approach including all known risk factors for very late stent thrombosis and predictors of increased bleeding risk [22]. In all ACS patients and possibly also in patients with more complex coronary lesions, there is a preference for the use of the more potent P2Y12 inhibitors, such as ticagrelor or prasugrel. As the prolongation of DAPT therapy from 1 to 3 years could prove to be harmful in specific patient subgroups, certainly those with a higher bleeding tendency and especially for patients already on oral anticoagulants, it is recommended to avoid implantation of the Absorb BVS in patients with a strict indication for oral anticoagulation.

\section{Conclusions}

The new 2017 recommendations downgrade and limit the use of current BVS to experienced centres within dedicated registries using the updated implantation protocol and advise the prolonged usage of DAPT in patients at high risk of ischaemic events. Patient should be well informed on the potential risks of the therapy and the only hypothetical long-term benefit. This recommendation is based on recent evidence from large randomised trials that implantation of BVS is associated with increased risk of adverse events, particularly increased risk of ScT and MI. In the near future the COMPARE ABSORB trial will report additional data regarding the appropriateness of BVS for PCI for specific lesion and patient subsets.

Conflict of interest B. Everaert, J. Koolen, P. van der Harst, P. den Heijer, J.P. Henriques, R. van der Schaaf, B. de Smet, S.H. Hofma, R. Diletti, A. Weevers, J. Hoorntje and P. Smits declare that they have no competing interests. R.J. van Geuns has received speakers fees from Abbott Vascular. J.J. Wykrzykowska received consultancy/ speaker fees from Abbott Vascular. The institutions Erasmus MC and Academic Medical Center, Amsterdam, received research grants from Abbott Vascular. The department of cardiology of the Medical Center Leeuwarden received unrestricted research grants from Abbott Vascular.

Open Access This article is distributed under the terms of the Creative Commons Attribution 4.0 International License (http:// creativecommons.org/licenses/by/4.0/), which permits unrestricted use, distribution, and reproduction in any medium, provided you give appropriate credit to the original author(s) and the source, provide a link to the Creative Commons license, and indicate if changes were made.

\section{References}

1. Stone GW, Moses JW, Ellis SG, et al. Safety and efficacy of sirolimus- and paclitaxel-eluting coronary stents. N Engl J Med. 2007;356:998-1008.

2. Simsek C, Magro M, Boersma E, et al. The unrestricted use of sirolimus- and paclitaxel-eluting stents results in better clinical outcomes during 6-year follow-up than bare-metal stents: an analysis 
of the RESEARCH (Rapamycin-Eluting Stent Evaluated At Rotterdam Cardiology Hospital) and T-SEARCH (Taxus-Stent Evaluated At Rotterdam Cardiology Hospital) registries. JACC Cardiovasc Interv. 2010;3:1051-8.

3. Everaert B, Felix C, Koolen J, et al. Appropriate use of bioresorbable vascular scaffolds in percutaneous coronary interventions: a recommendation from experienced users: a position statement on the use of bioresorbable vascular scaffolds in the Netherlands. Neth Heart J. 2015;23:161-5.

4. Serruys PW, Chevalier B, Dudek D, et al. A bioresorbable everolimus-eluting scaffold versus a metallic everolimus-eluting stent for ischaemic heart disease caused by de-novo native coronary artery lesions (ABSORB II): an interim 1-year analysis of clinical and procedural secondary outcomes from a randomised controlled trial. Lancet. 2015;385:43-54.

5. Ellis SG, Kereiakes DJ, Metzger DC, et al. Everolimus-eluting Bioresorbable scaffolds for coronary artery disease. N Engl J Med. 2015;373:1905-15.

6. Gao R, Yang Y, Han Y, et al. Bioresorbable vascular scaffolds versus metallic stents in patients with coronary artery disease: aBSORB China trial. J Am Coll Cardiol. 2015;66:2298-309.

7. Kimura T, Kozuma K, Tanabe K, et al. A randomized trial evaluating everolimus-eluting Absorb bioresorbable scaffolds vs. everolimus-eluting metallic stents in patients with coronary artery disease: ABSORB Japan. Eur Heart J. 2015;36:3332-42.

8. Stone GW, Gao R, Kimura T, et al. 1-year outcomes with the Absorb bioresorbable scaffold in patients with coronary artery disease: a patient-level, pooled meta-analysis. Lancet. 2016;387:1277-89.

9. Serruys PW, Ormiston J, van Geuns RJ, et al. A polylactide bioresorbable scaffold eluting everolimus for treatment of coronary stenosis: 5-year follow-up. J Am Coll Cardiol. 2016;67:766-76.

10. Serruys PW, Chevalier B, Sotomi Y, et al. Comparison of an everolimus-eluting bioresorbable scaffold with an everolimuseluting metallic stent for the treatment of coronary artery stenosis (ABSORB II): a 3 year, randomised, controlled, single-blind, multicentre clinical trial. Lancet. 2016;388:2479-91.

11. Ellis SG, Kereiakes DJ, Stone GW. Everolimus-eluting bioresorbable vascular scaffolds in patients with coronary artery disease: ABSORB III trial 2-year results. Presented ACC, Washington DC, 17. - 19. March. 2017.

12. Stone GW. Impact of technique on early and late outcomes following coronary bioresorbable scaffold implantation: analysis from the ABSORB trials. Presented CRT. 2017.

13. Ellis SG, Kereiakes DJ, Stone GW. Everolimus-eluting bioresorbable vascular scaffolds in patients with coronary artery disease: a deep dive into ABSORB BVS 2-year outcomes. Presented ACC. 2017.

14. Felix C, Fam JM, Diletti R, et al. Mid- to long-term clinical outcomes of patients treated with the everolimus-eluting bioresorbable vascular scaffold. The BVS expand study. JACC Cardiovasc Interv. 2016;9:1652-63.

15. Tamburino C, Capranzano P, Gori T, et al. 1-year outcomes of everolimus-eluting bioresorbable scaffolds versus everolimuseluting stents: a propensity-matched comparison of the GHOSTEU and XIENCE V USA registries. JACC Cardiovasc Interv. 2016;9:440-9.

16. Wykrzykowska JJ, Kraak RP, Hofma SH, et al. Bioresorbable scaffolds versus metallic stents in routine PCI. N Engl J Med. 2017; doi:10.1056/NEJMoa1614954.

17. Ellis SG, Gori T, Steffenino G. et al. Clinical, angiographic and procedural correlates of absorb scaffold thrombosis - an analysis of 105 cases (a multicenter report). Presented at ACC. 2017.

18. Naganuma T, Colombo A, Lesiak M, et al. Bioresorbable vascular scaffold use for coronary bifurcation lesions: a substudy from GHOST EU registry. Catheter Cardiovasc Interv. 2017;89:47-56.
19. Geraci S, Kawamoto H, Caramanno G, et al. Bioresorbable everolimus-eluting vascular scaffold for long coronary lesions: a subanalysis of the international, multicenter GHOST-EU registry. JACC Cardiovasc Interv. 2017;10:560-8.

20. Ortega-Paz L, Capodanno D, Giacchi G, et al. Impact of overlapping on 1-year clinical outcomes in patients undergoing everolimus-eluting bioresorbable scaffolds implantation in routine clinical practice: Insights from the European multicenter GHOST-EU registry. Catheter Cardiovasc Interv. 2017;89:812-8.

21. Schnorbus B, Wiebe J, Capodanno D, et al. 12 months outcomes after bioresorbable vascular scaffold implantation in patients with acute coronary syndromes. data from the European multicentre GHOST-EU extended registry. EuroIntervention. 2017; doi:10. 4244/EIJ-D-16-00568.

22. Sabaté M, Windecker S, Iñiguez A, et al. Everolimus-eluting bioresorbable stent vs. durable polymer everolimus-eluting metallic stent in patients with ST-segment elevation myocardial infarction: results of the randomized ABSORB ST-segment elevation myocardial infarction-TROFI II trial. Eur Heart J. 2016;37:229-40.

23. Fam JM, Ojeda S, Garbo R, et al. Everolimus eluting bioresorbable scaffold for treatment of complex chronic total occlusions. EuroIntervention. 2017; doi:10.4244/EIJ-D-16-00253.

24. Gori T, Wiebe J, Capodanno D, et al. Early and midterm outcomes of bioresorbable vascular scaffolds for ostial coronary lesions: insights from the GHOST-EU registry. EuroIntervention. 2016;12:e550-e6.

25. Wu C, Camacho FT, King SB, et al. Risk stratification for long-term mortality after percutaneous coronary intervention. Circ Cardiovasc Interv. 2014;7:80-7.

26. Puricel S, Cuculi F, Weissner M, et al. Bioresorbable coronary scaffold thrombosis: multicenter comprehensive analysis of clinical presentation, mechanisms, and predictors. J Am Coll Cardiol. 2016;67:921-31.

27. Allahwala UK, Cockburn JA, Shaw E, et al. Clinical utility of optical coherence tomography (OCT) in the optimisation of absorb bioresorbable vascular scaffold deployment during percutaneous coronary intervention. EuroIntervention. 2015;10:1154-9.

28. Windecker S, Kolh P, Alfonso F, et al. ESC/EACTS Guidelines on myocardial revascularization: The Task Force on Myocardial Revascularization of the European Society of Cardiology (ESC) and the European Association for Cardio-Thoracic Surgery (EACTS). Developed with the special contribution of the European Association of Percutaneous Cardiovascular Interventions (EAPCI). Eur Heart J. 2014;35:2541-619.

29. Wiviott SD, Braunwald E, McCabe $\mathrm{CH}$, et al. Prasugrel versus clopidogrel in patients with acute coronary syndromes. N Engl J Med. 2007;357:2001-15.

30. Wallentin L, Becker RC, Budaj A, et al. Ticagrelor versus clopidogrel in patients with acute coronary syndromes. N Engl J Med. 2009;361:1045-57.

31. Colombo A, Chieffo A, Frasheri A, et al. Second generation drugeluting stents implantation followed by six versus twelve-month - dual antiplatelet therapy - the SECURITY randomized clinical trial. J Am Coll Cardiol. 2014;64:2086-97.

32. Tarantini G, Nai Fovino L, Tellaroli P, et al. Optimal duration of dual antiplatelet therapy after second-generation drug-eluting stent implantation in patients with diabetes: The SECURITY (SecondGeneration Drug-Eluting Stent Implantation Followed By Six- Versus Twelve-Month Dual Antiplatelet Therapy)-diabetes substudy. Int J Cardiol. 2016;207:168-76.

33. Mauri L, Kereiakes DJ, Yeh RW, et al. Twelve or 30 months of dual antiplatelet therapy after drug-eluting stents. New Engl J Med. 2014;371:2155-66.

34. Yeh RW, Secemsky EA, Kereiakes DJ, et al. Development and validation of a prediction rule for benefit and harm of dual antiplatelet 
therapy beyond 1 year after percutaneous coronary intervention. JAMA. 2016;315:1735-49.

35. Bonaca MP, Bhatt DL, Cohen M, et al. Long-term use of ticagrelor in patients with prior myocardial infarction. N Engl J Med. 2015;372:1791-800.

36. Udell JA, Bonaca MP, Collet JP, et al. Long-term dual antiplatelet therapy for secondary prevention of cardiovascular events in the subgroup of patients with previous myocardial infarction: a collaborative meta-analysis of randomized trials. Eur Heart J. 2016;37:390-9.

37. Bittl JA, Baber U, Bradley SM, Wijeysundera DN. Duration of dual antiplatelet therapy: a systematic review for the 2016 ACC/AHA guideline focused update on duration of dual antiplatelet therapy in patients with coronary artery disease: a report of the American College of Cardiology/American Heart Association task force on clinical practice guidelines. Circulation. 2016;134:e156-78. 